\title{
Analytical Centrifugation
}

National Cancer Institute

\section{Source}

National Cancer Institute. Analytical Centrifugation. NCI Thesaurus. Code C18797.

A centrifug ation technique that utilizes photographic capability to determine the time it takes a particle to travel through a centrifuge cell under standard conditions. 